\title{
HSF1 acetylation decreases its transcriptional activity and enhances glucolipotoxicity-induced apoptosis in rat and human beta cells
}

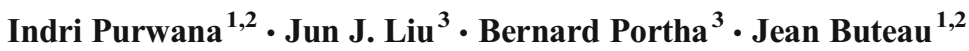

Received: 18 January 2017 / Accepted: 13 April 2017 /Published online: 25 May 2017

(C) Springer-Verlag Berlin Heidelberg 2017

\begin{abstract}
Aims/hypothesis Heat shock factor protein 1 (HSF1) is a transcription factor that regulates the expression of key molecular chaperones, thereby orchestrating the cellular response to stress. This system was recently implicated in the control of insulin sensitivity and is therefore being scrutinised as a novel therapeutic avenue for type 2 diabetes. However, the regulation and biological actions of HSF1 in beta cells remain elusive. Herein, we sought to investigate the regulation of HSF1 in pancreatic beta cells and to study its potential role in cell survival.

Methods We exposed human islets and beta cell lines to glucolipotoxicity and thapsigargin. HSF1 activity was evaluated by gel shift assay. HSF1 acetylation and interaction with the protein acetylase cAMP response element binding protein (CBP) were investigated by western blot. We measured the expression of HSF1 and its canonical targets in islets from Goto-Kakizaki (GK) rat models of diabetes and delineated the effects of HSF 1 acetylation using mutants mimicking constitutive acetylation and deacetylation of the protein.

Results Glucolipotoxicity promoted HSF1 acetylation and interaction with CBP. Glucolipotoxicity-induced HSF1 acetylation inhibited HSF1 DNA binding activity and decreased the
\end{abstract}

Jean Buteau

jbuteau@ualberta.ca

1 Alberta Diabetes Institute, University of Alberta, Li Ka Shing Centre, Edmonton, AB T6G 2E1, Canada

2 Department of Agricultural, Food and Nutritional Science, University of Alberta, Edmonton, AB, Canada

3 Laboratoire Biologie et Pathologie du Pancréas Endocrine (B2PE), Unité Biologie Fonctionnelle et Adaptive (BFA), Centre national de la recherche scientifique (CNRS), Unité mixte de recherche (UMR) 8251, Université Paris Diderot, Sorbonne-Paris-Cité, Paris, France expression of its target genes. Restoration of HSF1 activity in beta cells prevented glucolipotoxicity-induced endoplasmic reticulum stress and apoptosis. However, overexpression of a mutant protein $(\mathrm{K} 80 \mathrm{Q})$ mimicking constitutive acetylation of HSF1 failed to confer protection against glucolipotoxicity. Finally, we showed that expression of HSF1 and its target genes were altered in islets from diabetic GK rats, suggesting that this pathway could participate in the pathophysiology of diabetes and constitutes a potential site for therapeutic intervention.

Conclusions/interpretation Our results unravel a new mechanism by which HSF1 inhibition is required for glucolipotoxicityinduced beta cell apoptosis. Restoring HSF1 activity may represent a novel strategy for the maintenance of a functional beta cell mass. Our study supports the therapeutic potential of HSF1/heat shock protein-targeting agents in diabetes treatment.

Keywords Apoptosis · Betacell · Chaperones · Diabetes · ER stress · GK rat · Glucolipotoxicity · Goto-Kakizaki · Heat shock $\cdot$ HSF1

$\begin{array}{ll}\text { Abbreviations } \\ \text { CBP } & \text { cAMP response element binding protein } \\ \text { CHOP } & \text { CCAAT/enhancer-binding protein } \\ & \text { homologous protein } \\ \text { eIF2 } \alpha & \text { Eukaryotic initiation factor 2 subunit } \alpha \\ \text { ER } & \text { Endoplasmic reticulum } \\ \text { GFP } & \text { Green fluorescent protein } \\ \text { GK } & \text { Goto-Kakizaki } \\ \text { HSE } & \text { Heat shock element } \\ \text { HSF1 } & \text { Heat shock factor protein 1 } \\ \text { HSP } & \text { Heat shock protein } \\ \text { KRBH } & \text { Krebs-Ringer bicarbonate HEPES buffer } \\ \text { qChIP } & \text { Quantitative chromatin immunoprecipitation }\end{array}$


qPCR Quantitative PCR

SIRT1 Sirtuin 1

siRNA Small interfering RNA

UPR Unfolding protein response

\section{Introduction}

Type 2 diabetes results from the progressive deterioration of beta cell mass and function [1]. Indeed, a study of 82 individuals undergoing pancreatic surgery demonstrated that postchallenge glycaemia inversely correlated with beta cell mass [2]. The authors showed that a $21 \%$ reduction in beta cell mass was sufficient to cause impaired fasting glucose, and type 2 diabetes arose when beta cell mass was further reduced. These observations dovetail with findings from a previous study in which pancreases from cadaveric donors with type 2 diabetes displayed a 50\% reduction in beta cell mass when compared with pancreases from individuals without diabetes [3].

Pancreatic beta cells are destroyed by apoptosis [4] in response to a variety of environmental cues, including chronic exposure to hyperglycaemia and hyperlipidaemia, a condition termed 'glucolipotoxicity' [5]. Glucolipotoxicity imposes a metabolic stress, which can provoke mitochondrial dysfunction [6] and endoplasmic reticulum (ER) stress [7, 8]. The subsequent accumulation of misfolded proteins initiates an adaptive mechanism known as the unfolding protein response (UPR) to alleviate the burden of ER stress. This includes attenuation of protein synthesis and upregulation of molecular chaperones, the role of which is to increase ER folding capacity [9]. When ER stress is too severe and protein misfolding is not resolved, the cells enter apoptosis.

Heat shock factor protein 1 (HSF1) is a transcription factor that orchestrates the UPR. Indeed, HSF1 binds heat shock elements (HSEs) in the promoter regions of heat shock proteins (HSPs), which act as molecular chaperones to target damaged proteins for proteosomal degradation [10]. Through its key role in the regulation of the UPR, HSF1 coordinates the cellular response to diverse environmental stresses that extend well beyond heat shock (reviewed in [11]).

HSF1 transcriptional activity is regulated by various posttranslational modifications, including stress-inducible serine phosphorylation, phosphorylation-mediated sumoylation and lysine acetylation. Importantly, acetylation of an evolutionarily conserved lysine in position 80 (K80) facilitates its release from DNA, thereby repressing the expression of HSPs and attenuating the heat shock response [12].

Effectors of the heat shock response, such as HSPA1A, have been linked to insulin resistance and have thus garnered recent interest. Indeed, HSPA1A expression was found to be significantly reduced in muscle biopsies from individuals with type 2 diabetes [13]. Interestingly, overexpression of HSPA1A in skeletal muscle of mice prevented the development of insulin resistance in obese mice [14]. Moreover, $o b / o b$ mice treated with a small-molecule activator of HSPA1A, BGP-15, displayed improvements in fasting glucose and glucose disposal rate during hyperinsulinaemic-euglycaemic clamps [14]. Collectively, these studies suggest that HSPs could be therapeutically harnessed in peripheral tissues to improve insulin sensitivity. However, the regulation and biological roles of HSF1/HSPs have been less studied in pancreatic beta cells and remain elusive.

In this study, we sought to investigate the regulation of HSF1 in beta cells exposed to metabolic stress and to test the hypothesis that HSF1 could constitute a molecular target for the prevention of pancreatic beta cell death.

\section{Methods}

Human islets Human islets were purchased from the Alberta Diabetes Institute Islet Core at the University of Alberta with the assistance of the Human Organ Procurement and Exchange Program and the Trillium Gift of Life Network who provide donor pancreases for research. Islets from healthy lean donors aged 48-74 years were used. Islets were cultured in $11 \mathrm{mmol} / \mathrm{l}$ glucose DMEM medium supplemented with $10 \mathrm{mmol} / \mathrm{l} \mathrm{HEPES}$, $10 \%$ (vol./vol.) heat-inactivated fetal bovine serum, $2 \mathrm{mmol} / \mathrm{l} \mathrm{L}-$ glutamine, $1 \mathrm{mmol} / \mathrm{l}$ sodium pyruvate, $1 \%$ (vol./vol.) penicillinstreptomycin and $50 \mu \mathrm{mol} / / \beta$-mercaptoethanol at $37^{\circ} \mathrm{C}$ in a humidified $5 \% \mathrm{CO}_{2}$ atmosphere. Our study was approved by the Human Research Ethics Board at the University of Alberta.

Cell culture and treatment INS1832/13 cells (from C. Newgard, Duke University, Durham, NC, USA) (passage 50-75) were grown in RPMI 1640 medium supplemented with $10 \%$ (vol./vol.) heat-inactivated fetal calf serum, $2 \mathrm{mmol} / \mathrm{l} \mathrm{L}$-glutamine, $10 \mathrm{mmol} / \mathrm{l} \mathrm{HEPES}, 1 \mathrm{mmol} / \mathrm{l}$ sodium pyruvate and $50 \mu \mathrm{mol} / 1 \beta$-mercaptoethanol at $37^{\circ} \mathrm{C}$ in a humidified 5\% $\mathrm{CO}_{2}$ atmosphere. Mycoplasma testing was performed to ensure negative status. The authenticity of the cell line was verified through maintenance of glucose-stimulated insulin secretion. Cells were treated with $25 \mathrm{mmol} / \mathrm{l}$ glucose and $0.4 \mathrm{mmol} / \mathrm{l}$ palmitate (glucolipotoxicity) for various time periods (up to $48 \mathrm{~h}$ ) or $1 \mu \mathrm{mol} / \mathrm{l}$ thapsigargin (for up to $7 \mathrm{~h}$ ).

Animals Adult male (10 weeks old) and neonate ( 7 days old) Goto-Kakizaki (GK) rat models of diabetes were obtained from the Paris colony (GK/Par), maintained at the University Paris Diderot animal core. Age-matched Wistar rats were also from our local colony. Our study was in accordance with accepted standards of animal care as established in the French National Center for Scientific Research guidelines and approved by our institution. Animals were housed in a temperature-controlled environment, on a $12 \mathrm{~h}$ light/dark cycle with ad libitum access to food and water. 
Electrophoretic mobility shift assay An electrophoretic mobility shift assay (EMSA) was performed as described elsewhere [15]. Briefly, $3 \mu \mathrm{g}$ of nuclear protein extracts were incubated with a biotin-labelled probe containing the proximal heat shock responsive element of the Hspala promoter [16] for $30 \mathrm{~min}$ at room temperature. Samples were then loaded on $6 \%$ non-denaturing polyacrylamide gels and revealed with streptavidin - horseradish peroxidase (HRP) (Thermo Fisher Scientific, Waltham, MA, USA). Competition with 50-fold excess of unlabelled oligonucleotide or anti-HSF1 antibody (Millipore, Etobicoke, ON, Canada) was performed to demonstrate specificity of the binding.

Quantitative chromatin immunoprecipitation Quantitative chromatin immunoprecipitation (qChIP) was performed using a commercial kit (Millipore, Etobicoke, ON, Canada). In brief, cells were fixed and extracts were subjected to shearing by sonication. One-tenth of the sheared DNA was retained to evaluate fragment sizes and used as input. Then, HSF1 complexes were immunoprecipitated, washed and reverse crosslinked. Bound DNA was purified on Oligonucleotide Purification Columns (Qiagen, Toronto, ON, Canada) and amplified by quantitative PCR (qPCR). The primers were designed to amplify a $97 \mathrm{bp}$ region upstream of the transcription start site, which contains three putative HSEs (Table 1). PCR products were loaded on a gel to confirm that a single band was present. Sample values were normalised to their respective input.

Immunoprecipitation and western blot Cells were lysed (50 mmol/1 Tris, $300 \mathrm{mmol} / \mathrm{l} \mathrm{NaCl}, 1 \%$ Triton $\mathrm{X}$ ), scraped and centrifuged $\left(10,000 \mathrm{~g}\right.$ at $\left.4{ }^{\circ} \mathrm{C}\right)$ for $10 \mathrm{~min}$. Proteins were quantified by BCA assay (Roche Applied Science, Laval, QC, Canada). HSF1 protein complexes were immunoprecipitated from $1 \mathrm{mg}$ of protein and subjected to SDS-PAGE on $8 \%$ or $10 \%$ polyacrylamide gels. Whole-cell extracts $(15 \mu \mathrm{g})$ were used as input. We used the following antibodies: rabbit antiHSF1, rabbit anti-SIRT1, rabbit anti-phospho-eukaryotic initiation factor 2 subunit $\alpha$ (eIF2 $\alpha)$ and rabbit anti-acetylatedlysine (all from Millipore, Etobicoke, ON, Canada and used a dilution of 1:1000), rabbit anti-CBP, rabbit anti-CCAAT/enhancer-binding protein homologous protein (CHOP) and mouse anti-actin (Santa Cruz, Dallas, TX, USA; 1:200).

Plasmids HSF1-green fluorescent protein (GFP) (Plasmid 32538) was purchased from Addgene (Cambridge, MA, USA). All other HSF1 vectors were kind gifts from S. Westerheide (University of South Florida, FL, USA) [12]. Sirtuin 1 (SIRT1) plasmid was described previously [17]. cAMP response element binding protein (CBP) vector was from Addgene (Plasmid 32908).
Table 1 List of primers used for real-time qPCR and qChIP

\begin{tabular}{ll}
\hline Gene & Primer \\
\hline qPCR & \\
Rat Hspala & F: 5'-CAAGATCACCATCACCAACG-3' \\
& R: 5'-GCTGATCTTGCCCTTGAGAC-3' \\
Rat Dnajb1 & F: 5'-TAGCTTTCCGATGGGTATGG-3' \\
& R: 5'-CGCTTGTGGGAGATTTCAT-3' \\
Rat Usp24 & F: 5'-GAGATTCCATCCCGTCAGAA-3' \\
& R: 5'-ATGTTGGCATTGTCTGTCCA-3' \\
Rat Hhex & F: 5'-GAGGTTTTCCAACGACCAGA-3' \\
& R: 5'-GGATTCTCCTGCTTCAGTCG-3' \\
Rat Hsf1 & F: 5'-TCTCACTGGTGCAGTCGAAC-3' \\
Rat actin & R: 5'-GCTGTAGGCTGGAGATGGAG-3' \\
& F: 5'-GTGCCCATCTATGAGGGTTACGCG-3' \\
Human HSPA1A & F: 5'-GGAACCGCTCATTGCCGATAGTG-3' \\
& R: 5'-CACCTTGCCGTGTTGGAA-3' \\
Human HSPA6 & F: 5'-GAGGTGGAGAGGATGGTTCA-3' \\
& R: 5'-TGTCCTCTTCGGGAATCTTG-3' \\
Human DNAJB1 & F: 5'-CTTTCCCCAAGGAAGGAGAC-3' \\
& R: 5'-ATACGACGGGTATCGTCCTG-3' \\
Human actin & F: 5'-AGAAAATCTGGCACCACACC-3' \\
qChIP & R: 5'-GGGGTGTTGAAGGTCTCAAA-3' \\
& F: 5'-CCTTAAACTCCCTCCCTGGTCT-3' \\
& R: 5'-GTAGCGGTCTCTGTATGTGAAGCT-3' \\
\hline
\end{tabular}

Transfection DNA vectors were introduced into INS832/13 cells by nucleofection (Lonza, Mississauga, ON, Canada) following manufacturer's protocol. Human islet cells were transduced using Lipofectamine 2000 (Thermo Fisher Scientific, Waltham, MA). Hsf1 small interfering RNAs (siRNAs) (Locus ID 79245, siRNA ID numbers s135142, s135144, s222830, henceforth referred to as siHSF1 (1), siHSF1 (2) and siHSF1 (3), respectively) were purchased from Thermo Fisher Scientific and transfected using Lipofectamine RNAiMax.

Apoptosis Apoptosis was assessed by TUNEL assay using a TMR red in situ cell death detection kit (Roche Applied Science), as described elsewhere [18]. In brief, INS832/13 cells or islet cells were seeded onto polylysine-coated coverslips, fixed in paraformaldehyde, permeabilised with $0.1 \%$ (vol./vol.) Triton $\mathrm{X}-100$ in PBS and incubated for $1 \mathrm{~h}$ with a TUNEL reaction mix followed by DAPI staining. The coverslips were then washed in PBS and mounted with Fluoromount G (Thermo Fisher Scientific, Waltham, MA, USA). The fluorescence was visualised under a fluorescence microscope at $400 \times$ magnification. At least 500 cells were analysed for each experimental condition. The results are expressed as percentage of TUNELpositive cells (red/blue nuclei).

Gene expression Total RNA was extracted from rat islets using the RNeasy mini kit (Qiagen, Les Ulis, France). cDNA of each RNA sample was synthesised with Moloney 
Murine Leukemia Virus (M-MLV) reverse transcriptase (Thermo Fisher Scientific, Villebon, France) using random hexamer primers. mRNA transcript levels of four housekeeping genes (encoding ribosomal protein L19 [Rpl19], TATAbox binding protein $[T b p]$, cyclophilin A and $18 \mathrm{~S}$ ) were assayed. Since similar results were obtained with the four housekeeping genes, only cyclophilin A was retained for normalisation of other transcripts. Human islet and INS1 cell line RNA was extracted (RNeasy; Qiagen, Toronto, ON, Canada), reverse transcribed (Superscript RT II; Thermo Fisher Scientific, Waltham, MA, USA) and assayed by real-time qPCR (SYBR Green; Qiagen, Toronto, ON) according to the manufacturers' instructions. Results were normalised to actin. Primers are listed in Table 1.

$\mathrm{NAD}^{+} / \mathrm{NADH}$ ratio $\mathrm{NAD}^{+}$and $\mathrm{NADH}$ levels were measured in whole-cell extracts using a commercial kit from Biovision (Mountain View, CA, USA).

Insulin secretion and insulin content Cells were preincubated in Krebs-Ringer bicarbonate HEPES buffer (KRBH) medium containing $2 \mathrm{mmol} / \mathrm{l}$ glucose for $30 \mathrm{~min}$. Then, cells were incubated in KRBH medium containing $2 \mathrm{mmol} / \mathrm{l}$ glucose, $16 \mathrm{mmol} / \mathrm{l}$ glucose or $35 \mathrm{mmol} / \mathrm{l} \mathrm{KCl}$ for $45 \mathrm{~min}$. Media were collected and insulin concentrations were measured by ELISA (Alpco, Salem, NH, USA). Cells were lysed in acidic ethanol for determination of insulin content.

Statistical analysis Data are presented as mean \pm SEM. Statistical analyses were performed with GraphPad Prism 6 (GraphPad Software, La Jolla, CA, USA) using ANOVA followed by post hoc tests as appropriate. Statistical significance was declared when $p \leq 0.05$. The experimenters were not blind to group assignment and no data were omitted.

\section{Results}

\section{Glucolipotoxicity inhibits HSF1 activity via CBP-mediated} acetylation We first sought to explore the regulation of HSF1 in pancreatic beta cells in response to glucolipotoxicity, a condition of metabolic stress that provokes ER stress [19-21]) and is thought to mimic the hyperglycaemic and hyperlipidaemic environment associated with diabetes $[1,4]$.

We thus exposed INS cells to elevated concentrations of glucose $(25 \mathrm{mmol} / \mathrm{l})$ and palmitate $(0.4 \mathrm{mmol} / \mathrm{l}$, complexed to BSA $)$ for up to $24 \mathrm{~h}$ and measured p-eIF $2 \alpha$ as well as CHOP protein levels as markers of ER stress. Our results confirmed that glucolipotoxicity causes ER stress in beta cells, as illustrated by the sequential increase in the expression of p-eIF $2 \alpha$ and CHOP (Fig. 1a). Glucolipotoxicity induced a transient increase in p-eIF2a that preceded the increase in CHOP, which was maximal at $24 \mathrm{~h}$. Thapsigargin, used as a positive control of ER
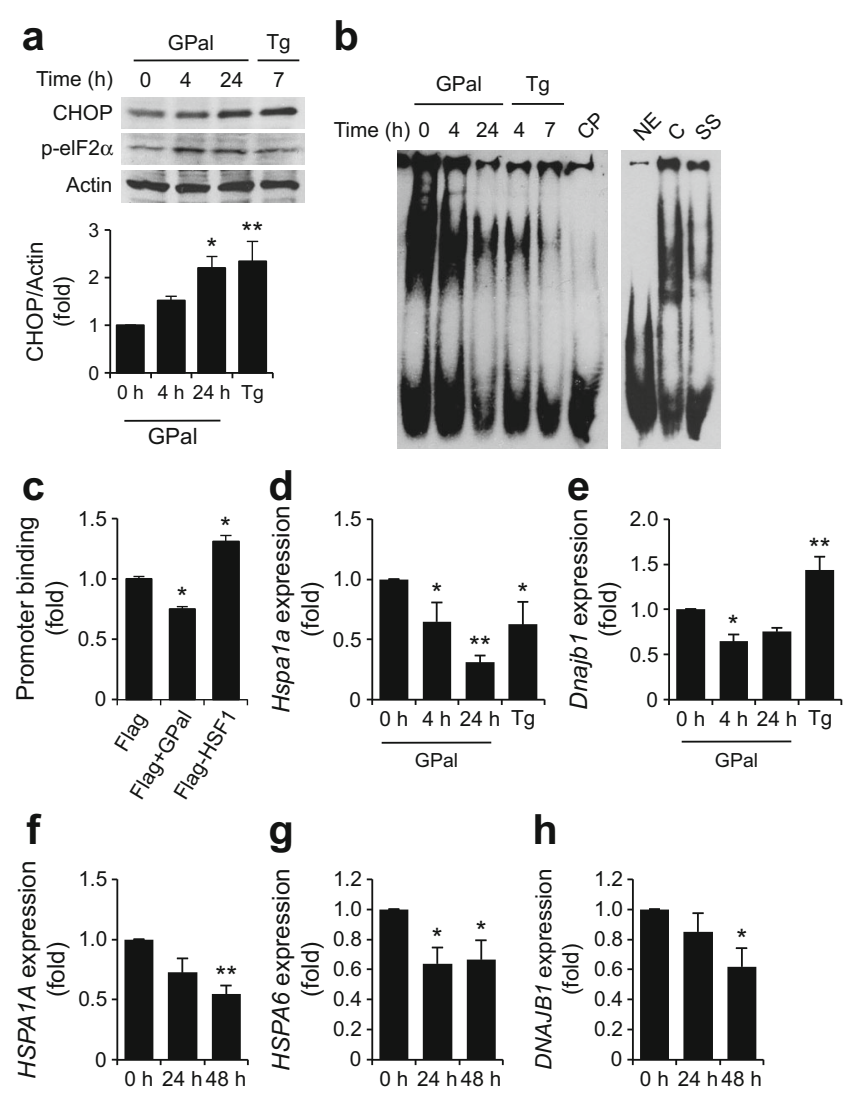

Fig. 1 Glucolipotoxicity reduces HSF1 activity. (a) Protein levels of the ER stress markers CHOP and p-eIF $2 \alpha$ were evaluated by western immunoblot in INS cells treated with $25 \mathrm{mmol} / \mathrm{l}$ glucose and $0.4 \mathrm{mmol} / \mathrm{l} \mathrm{pal}-$ mitate $(\mathrm{GPal})$ or $1 \mu \mathrm{mol} / \mathrm{l}$ thapsigargin $(\mathrm{Tg})$ for the indicated periods of time. Densitometric quantification of three independent experiments is shown for CHOP. (b) HSF1 DNA binding activity was evaluated by gel shift assay. Binding specificity was confirmed using a 50-fold excess of unlabelled probe (cold probe, CP) and an anti-HSF1 antibody (supershift, $\mathrm{SS}$ ). Representative images of at least three separate experiments are shown. C, control untreated cells; NE, no extract. (c) HSF1 binding to the Hspala promoter was measured by qChIP. Flag-HSF1 was used as a positive control. (d-h) Expression of (d) Hspala, (e) Dnajbl in INS cells and (f) HSPA1A, (g) HSPA6 and (h) DNAJB1 in human islets was measured by qPCR and normalised to actin. Results are shown as mean \pm SEM of three independent experiments. ${ }^{*} p<0.05$, $* * p<0.01$ vs non-treated control samples

stress, also increased CHOP protein levels. However, we did not detect an increase in p-eIF2a at the tested time point $(7 \mathrm{~h})$.

We then sought to investigate the effects of both glucolipotoxicity and thapsigargin-induced ER stress on HSF1 activity. We thus measured HSF1 DNA binding activity, in the absence or presence of ER stress, using a probe corresponding to the proximal region of the Hspala promoter, which contains an HSE sequence [16]. Figure $1 \mathrm{~b}$ shows that both glucolipotoxicity and thapsigargin caused a time-dependent decrease in the DNA binding activity of HSF1. This effect was not due to a reduction in HSF1 protein levels since neither glucolipotoxicity nor thapsigargin altered total HSF1 protein levels (as seen in Fig. 2c, d-d). qChIP experiments carried out in INS cells showed that glucolipotoxicity decreased HSF1 binding to the 

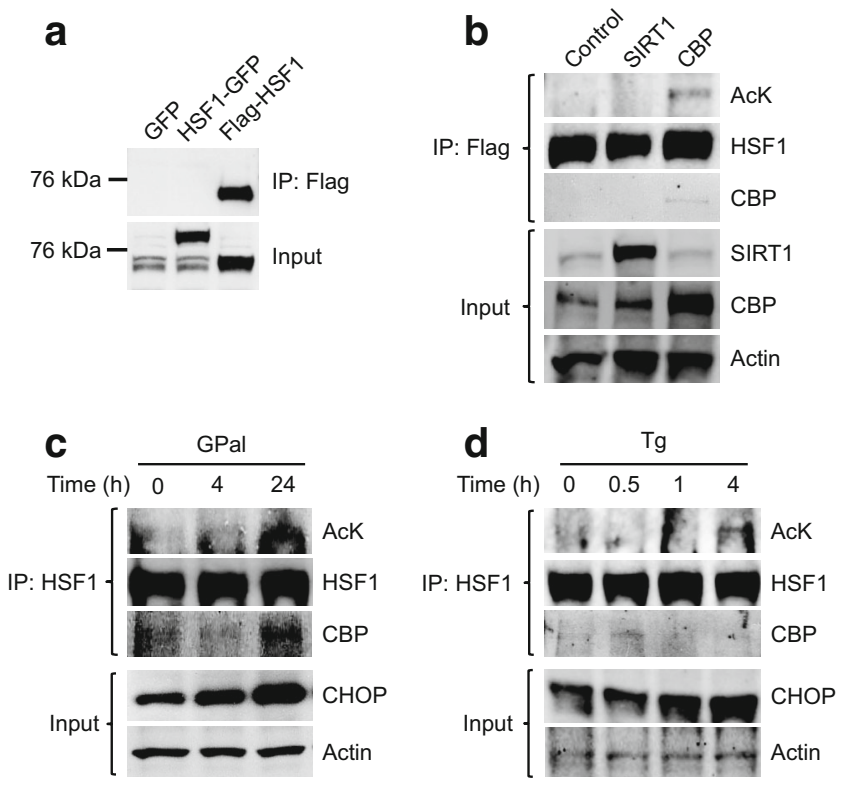

Fig. 2 Glucolipotoxicity induces HSF1 acetylation. (a) Validation of HSF1-expressing vectors. Cells were transfected with a GFP control vector or with either Flag- or GFP-tagged HSF1 plasmids for 24 h. 'IP: Flag': cell lysates were immunoprecipitated (IP) for Flag and immunoblotted for HSF1. 'Input': total lysates were probed for HSF1. (b) INS cells were transfected with HSF1-Flag along with empty (control), SIRT1 or CBP plasmids for $24 \mathrm{~h}$. HSF1 protein complexes were immunoprecipitated from cell lysates using an anti-Flag antibody followed by immunoblot against acetylated-lysine (AcK), HSF1 and CBP (IP: Flag). SIRT1 and CBP overexpression were verified in total cell lysates (Input). Actin was used as a loading control. (c) INS cells were subjected to glucolipotoxicity (GPal) or (d) treated with thapsigargin (Tg) for the indicated periods of time. HSF1 acetylation and binding to CBP was evaluated by western blot after immunoprecipitation of HSF1 protein complexes. HSF1 acetylation was correlated to CHOP protein levels in whole-cell extracts. Representative images of at least three separate experiments are shown

endogenous Hspala promoter by $25 \%$ whereas transfection of the cells with Flag-HSF1 increased binding by $40 \%$ (Fig. 1c). ER stress-mediated inhibition of HSF1 translated into reduced expression of its target genes, including Hspala and Dnajb1 in INS cells (Fig. 1d, e) and HSPA1A, DNAJB1 and HSPA6 in human islets (Fig. 1f-h). There are no known orthologues of HSPA6 in rats. Unexpectedly, thapsigargin increased Dnajb1 expression in INS cells. The cause of this discrepancy is unknown.

Taken together, these results indicate that environmental stress acutely inhibits HSF1 transcriptional activity, possibly via post-translational modification of the protein. HSF1 activity is indeed regulated via multiple post-translational modifications [22], including phosphorylation and acetylation. HSF1 acetylation is regulated by the antagonistic actions of CBP and SIRT1. Acetylation by CBP has been shown to maintain the protein in an inactive state, whereas SIRT1-dependent deacetylation maintains HSF1 in a competent DNA binding state [12].

We thus sought to investigate both HSF1 acetylation and interaction with $\mathrm{CBP}$ by western blot after immunoprecipitation of HSF1 protein complexes from whole-cell extracts. Figure 2a validates the molecular tools employed in our study. HSF1-GFP and Flag-HSF1 plasmids both induced a fivefold increase in HSF1 protein levels when compared with GFP control (Fig. 2a). Only Flag-HSF1 could be detected after immunoprecipitation with a Flag antibody. Figure 2b shows that in the basal situation HSF1 is mostly deacetylated and thus in an active state. However, overexpression of the acetyltransferase CBP caused HSF1 acetylation (Fig. 2b), demonstrating that HSF1 is a bona fide target of CBP in beta cells. SIRT1 overexpression did not affect HSF1 acetylation since it was already minimal. We next tested whether endogenous HSF1 could be regulated via its acetylation in response to environmental stress. Our results showed that both glucolipotoxicity (Fig. 2c) and thapsigargin-induced ER stress (Fig. 2d) provoked HSF1 acetylation. This effect was concomitant with an increased interaction with $\mathrm{CBP}$, which was apparent at $24 \mathrm{~h}$ and $0.5 \mathrm{~h}$, respectively.

Restoration of HSF1 activity prevents glucolipotoxicityinduced apoptosis To demonstrate a causal role of HSF1 in beta cell survival, we examined the consequences of HSF1 gain- and loss-of-function experiments, using overexpression and siRNA-mediated knockdown, respectively. We evaluated ER stress by measuring CHOP protein levels and apoptosis by TUNEL assay. Consistent with data shown in Fig. 1, glucolipotoxicity induced a twofold increase in CHOP protein levels. However, overexpression of HSF 1 blunted the glucolipotoxicity-induced CHOP expression (Fig. 3a), supporting our hypothesis that HSF1 inhibition was instrumental in causing ER stress. Moreover, HSF1 overexpression prevented glucolipotoxicity-induced apoptosis in INS cells (Fig. 3b, c) and human islet cells (Fig. 3d). We next examined whether loss of HSF1 would have the opposite effect and induce ER stress in beta cells. Hsfl gene silencing using three different siRNA sequences led to a $90 \%$ reduction in Hsfl mRNA levels in INS cells, as measured by qPCR (Fig. 3e). Hsfl knockdown translated into a $\sim 50 \%$ reduction in HSF1 protein levels (Fig. 3f) but it failed to upregulate $\mathrm{CHOP}$ protein levels per se, suggesting that HSF1 protein knockdown is not sufficient to cause ER stress in the absence of a positive stimulus.

Constitutively acetylated HSF1 lacks transcriptional activity and protective effects To elucidate the biological role of HSF 1 acetylation in beta cells, we overexpressed HSF1 mutants bearing mutations at lysine 80 to either mimic constitutive acetylation (K80Q) or deacetylation (K80R) of the protein. Indeed, a previous publication established that acetylation of K80 is critical for the DNA binding capacity of HSF1 [12].

To confirm that $\mathrm{K} 80$ represents a prominent acetylation site in our model, we first transfected INS cells with wild-type HSF1, K80Q-HSF1 or K80R-HSF1 and exposed the cells to glucolipotoxicity for $24 \mathrm{~h}$ to induce acetylation. We then measured HSF1 acetylation and interaction with CBP by western blot after immunoprecipitation of HSF1 complexes (Fig. 4a). 


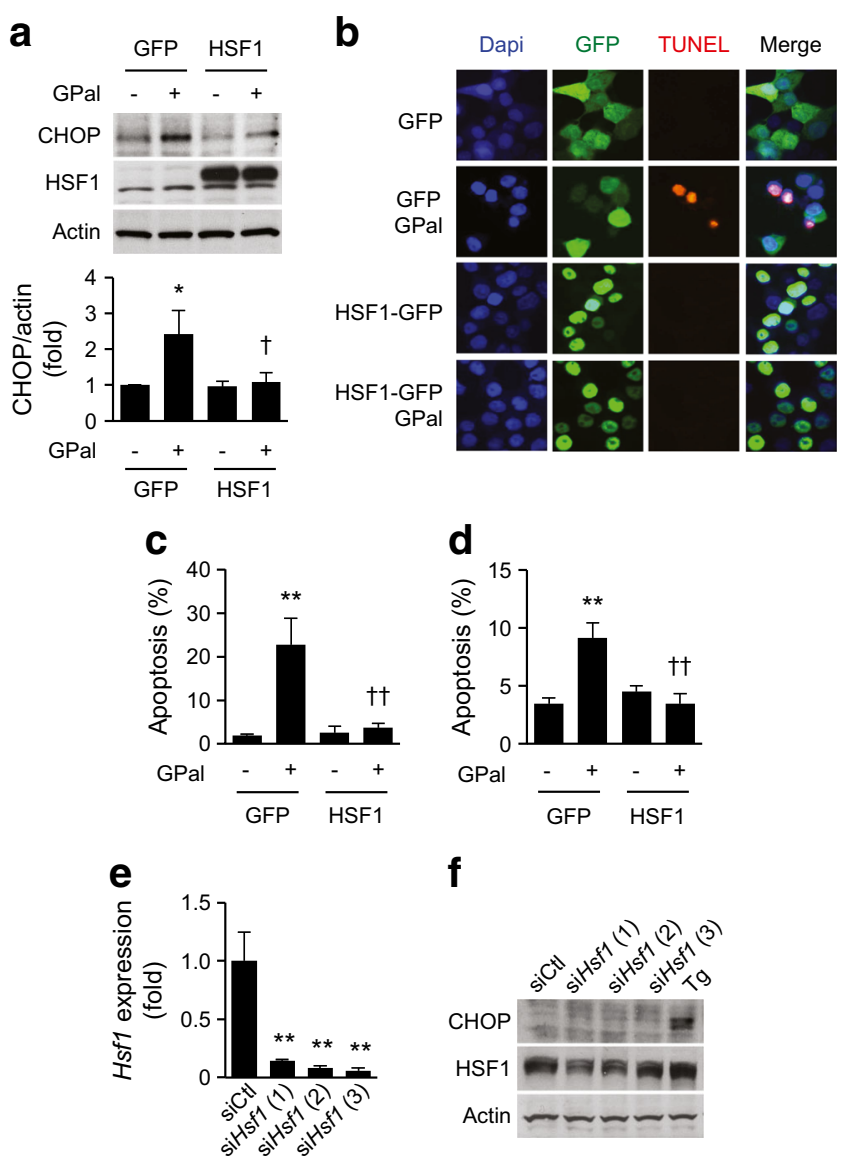

Fig. 3 Restoration of HSF1 activity prevents glucolipotoxicity-induced ER stress and apoptosis. (a) CHOP protein levels were evaluated by western blot in cells transfected with either a control GFP or HSF1GFP plasmid. Representative blots and quantification (relative to control [GFP, no glucolipotoxicity]) are shown. (b-d) Apoptosis was assessed by TUNEL assay in (b, c) INS cells and (d) human islet cells transfected with GFP or HSF1-GFP and treated in the absence or presence of glucolipotoxicity (GPal) for $24 \mathrm{~h}$. Representative images are shown in (b). Data are expressed as percentages of apoptotic nuclei (TUNEL-positive [red] cells/total number of nuclei [blue]). (e) Hsfl mRNA expression was examined by qPCR in INS cells transfected with either control siRNA (siCtl) or three different HSF1-specific siRNA sequences. Results are normalised to actin. (f) CHOP and HSF1 protein levels were examined by western blot in siRNA-transfected cells. Thapsigargin (Tg) was used as a positive control of ER stress. Results are mean \pm SEM of at least three independent experiments. $* p<0.05, * * p<0.01$ vs control (GFP, no glucolipotoxicity); ${ }^{\dagger} p<0.05,{ }^{\dagger \dagger} p<0.01$ vs GFP plasmid

As expected, acetylation was detected in cells bearing wildtype HSF1 but not in the mutants bearing a K80 substitution. This result confirms that $\mathrm{K} 80$ is a major acetylation site in HSF1. Consistently, we detected a decrease in CBP-HSF1 interactions in the mutants compared with cells expressing wild-type HSF1.

We next determined the consequences of HSF1 acetylation on its transcriptional activity and ability to promote beta cell survival. We thus measured the effects of wild-type HSF1, K80Q-HSF1 and K80R-HSF1 on the expression of HSF1's canonical target Hspala by qPCR. Glucolipotoxicity decreased Hspala expression in control cells by $\sim 50 \%$ and this effect was partially abrogated in cells overexpressing wild-type HSF1 and K80R-HSF1 (Fig. 4b). However, the acetylation-mimicking mutant K80Q-HSF1 failed to provide rescue from glucolipotoxicity-induced reduction in Hspala expression, supporting our hypothesis that acetylation of HSF1 at position K80 is critical for its transcriptional activity.

To further investigate the biological consequences of HSF1 acetylation, we measured CHOP protein levels by western blot, and apoptosis by TUNEL assay. Both wild-type HSF 1 and K80R-HSF1 overexpression prevented glucolipotoxicityinduced CHOP activation (Fig. 4c). However, K80Q-HSF1 was ineffective, consistent with its decreased transcriptional activity. Similarly, both wild-type HSF1 and K80R-HSF1 overexpression prevented glucolipotoxicity-induced cell death in INS1 cells (Fig. 4d, e) and in human islets (Fig. 4f). In INS cells, not only was K80Q-HSF1 unable to prevent apoptosis, it also slightly increased basal levels. Collectively, our data suggest that acetylation of HSF1 is regulated by environmental stress, decreases its transcriptional activity and is necessary for the induction of beta cell apoptosis.

Because protein acetylation is tightly linked to cellular $\mathrm{NAD}^{+}$, we sought to measure the effects of glucolipotoxicity on $\mathrm{NAD}^{+} / \mathrm{NADH}$ ratios (Fig. $4 \mathrm{~g}$ ). Our data show that glucolipotoxicity decreased the $\mathrm{NAD}^{+} / \mathrm{NADH}$ ratio in INS cells, thereby providing a potential molecular mechanism by which glucolipotoxic stress could regulate the antagonistic actions of CBP and SIRT1.

HSF 1 activity is altered in a rat model of type 2 diabetes To demonstrate the clinical relevance of our findings, we sought to demonstrate altered HSF1 expression and/or activity in an animal model of diabetes. We thus used islets isolated from 10-week-old diabetic GK rats, one of the best characterised animal models of spontaneous type 2 diabetes [23] and islets from control Wistar rats to measure the expression of $H s f l$ and some of its proposed transcriptional targets, namely Hspala, Dnajb1, Usp24 and Hhex [24, 25] (Fig. 5a). Our results show that $H s f 1$ mRNA levels were decreased by nearly $30 \%$ in GK islets compared with wild-type controls. In accordance, mRNA expression of both molecular chaperones Hspala and Dnajb1 was greatly reduced in GK islets. We also detected a small but significant reduction in the expression of the ubiquitin hydrolase Usp24. HSF1 has been suggested to act as a transcriptional repressor on the Hhex promoter, a gene that has been previously linked to type 2 diabetes. Consistently with the decrease in HSF1 expression, we detected a significant increase in Hhex expression in GK islets.

Importantly, none of these genes were found to be differentially expressed in neonatal (7-day-old) GK islets compared with age-matched control islets (Fig. 5b). This suggests that the altered expression of $H_{s f l}$ and its transcriptional targets in diabetic adult GK islets is not intrinsic but instead acquired, 

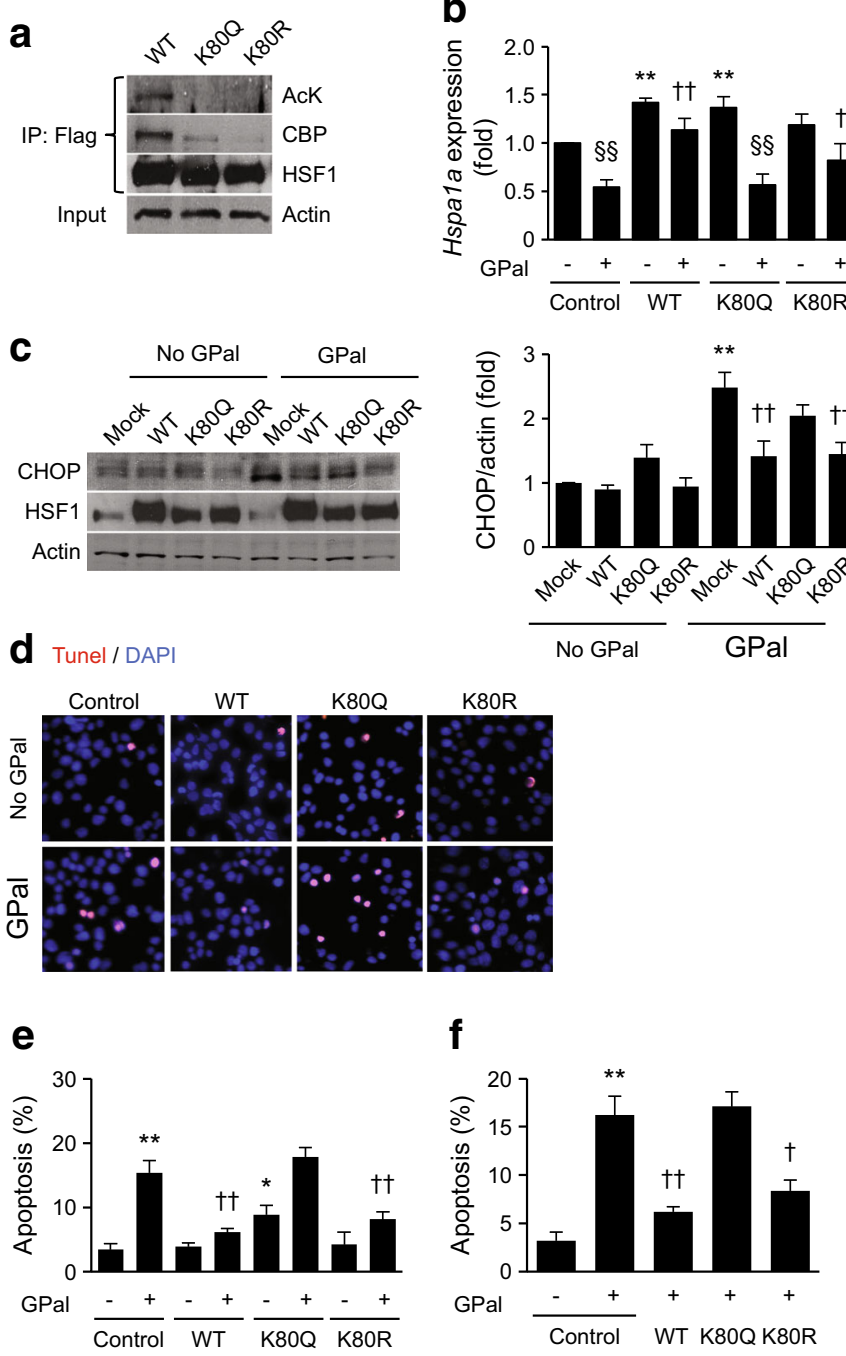
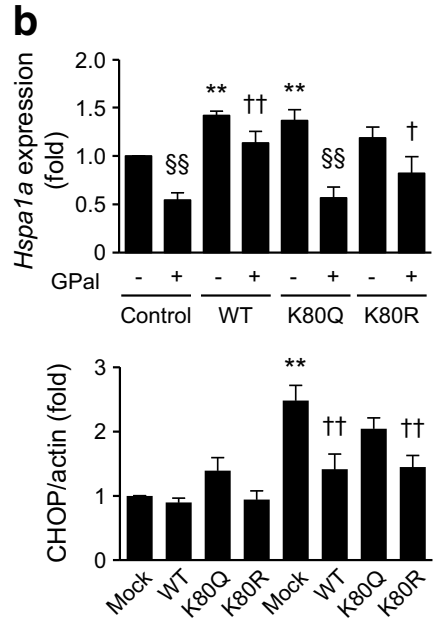

No GPal GPal

\section{g}

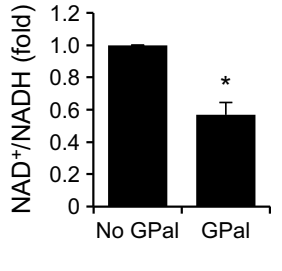

Fig. 4 Constitutively acetylated HSF1 lacks transcriptional activity and protective effects. (a) INS cells were transfected with wild-type (WT) HSF1 or mutants mimicking constitutive acetylation (K80Q) and deacetylation (K80R) of the protein, and exposed to glucolipotoxicity to induce acetylation of HSF1. All constructs were tagged with Flag. Then, HSF1 acetylation as well as binding to CBP was evaluated by western blot after immunoprecipitation of HSF1 complexes with an anti-Flag antibody (IP: Flag). (b) INS cells were transfected with an empty plasmid (control) or with WT-, K80Q- or K80R-HSF1, and subjected to glucolipotoxicity (GPal) for $24 \mathrm{~h}$. Hspala expression was measured by qPCR and normalised to actin. (c) CHOP protein levels were evaluated by

possibly in response to the progressive development of hyperglycaemia and diabetes-related metabolic conditions starting from the weaning period.

HSF1 does not restore the insulin secretory capacity of INS cells exposed to glucolipotoxicity Whereas our results suggest a critical role for HSF1 in beta cell death/survival, its potential action on beta cell function remains unknown. We thus measured insulin secretion in cells exposed or unexposed to glucolipotoxicity, in the presence and absence of HSF1 overexpression (Fig. 6a). As expected, cells western blot in cells treated as described above. Densitometric analysis of three separate experiment is shown. (d-f) Apoptosis was assessed by TUNEL assay in (d, e) INS cells and (f) human islet cells, transfected and treated with GPal as described above. Representative images are shown in (d). All nuclei were stained with DAPI (blue) and TUNELpositive nuclei stained red. (g) $\mathrm{NAD}^{+}$and NADH levels were determined in INS cells subjected to glucolipotoxicity for $24 \mathrm{~h}$. Data are shown as mean \pm SEM of three independent experiments. * $p<0.05$, ** $p<0.01 \mathrm{vs}$ control plasmid without GPal; ${ }^{\dagger} p<0.05,{ }^{\dagger \dagger} p<0.01$ vs control plasmid with GPal; ${ }^{\S} p<0.01$ vs corresponding GPal untreated condition. Representative blots of at least three experiments are shown

exposed to glucolipotoxic stress displayed impaired insulin exocytosis. Indeed, both glucose- and $\mathrm{KCl}$-induced insulin secretion was blunted by glucolipotoxicity. HSF1 overexpression failed to restore the secretory capacity of INS cells. Glucolipotoxicity also decreased insulin content by $27 \%$ in control cells (Fig. 6b), an effect that was prevented by HSF1 overexpression. We speculate that HSF1 target genes improve beta cell survival, and possibly insulin biosynthesis, because they alleviate the UPR. However, HSF1 target genes do not correct the metabolic/exocytosis defects induced by glucolipotoxicity. 

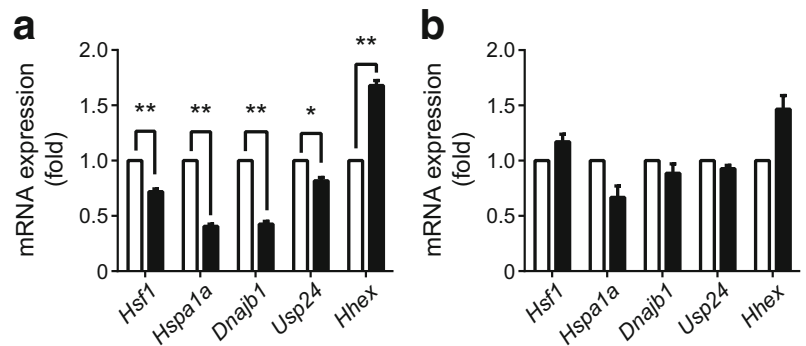

Fig. 5 Reduced expression of $H_{s f} 1$ and HSF1 targets in isolated islets from diabetic GK rats. Gene expression was measured by qPCR in islets isolated from (a) adult diabetic GK rats and (b) 7-day-old normoglycaemic neonates compared with control rats. White bars, control Wistar rats; black bars, GK rats. The results are shown as mean \pm SEM; control rats, $n=8$, GK rats, $n=6$. * $p<0.05$, ** $p<0.01$ vs control

Altogether, our results support a model (Fig. 7) in which decreased HSF1 activity precipitates beta cell demise and participates in the progression of type 2 diabetes.

\section{Discussion}

The transcription factor HSF1 is mostly known for its role in orchestrating the heat shock response. However, this system plays a fundamental role in providing protection against numerous other cellular stresses, including metabolic perturbations [26]. Indeed, HSPA1A mRNA expression was found to be decreased in muscle biopsies from individuals with type 2 diabetes and seemed to tightly correlate with insulin-stimulated glucose uptake during hyperinsulinaemic-euglycaemic clamps [13]. Moreover, manipulation of HSP expression in metabolic tissues has been reported to stimulate skeletal muscle oxidation and improve insulin sensitivity [27]. Consequently, effectors of the heat shock response are currently being investigated as potential sites of intervention for the prevention/treatment of type 2 diabetes. Small-molecule inducers of HSPA1A are currently
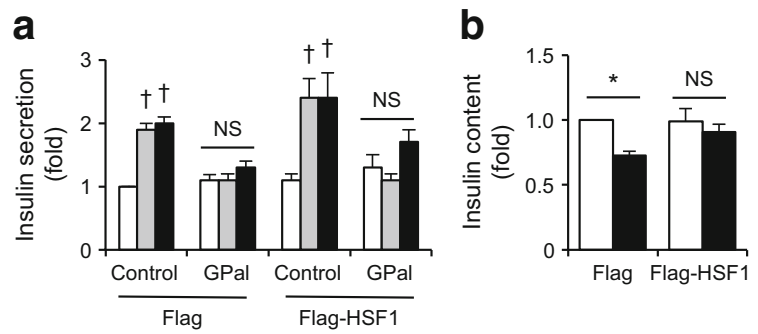

Fig. 6 HSF1 fails to restore insulin secretion in glucolipotoxicity. Cells were transfected with either Flag or Flag-HSF1. The next day they were exposed to glucolipotoxicity (GPal) for $24 \mathrm{~h}$. Then, (a) insulin secretion was measured in cells treated with $2 \mathrm{mmol} / 1$ glucose (white bars), $16 \mathrm{mmol} / 1$ glucose (grey bars) or $35 \mathrm{mmol} / 1 \mathrm{KCl}$ (black bars) and cells were lysed for determination of (b) insulin content (white bars, control [no glucolipotoxicity]; black bars, glucolipotoxicity). Insulin secretion data were normalised to insulin content. The results are shown as mean \pm SEM of three independent experiments, each comprising duplicates. $* p<0.05$ vs control; ${ }^{\dagger} p<0.05$ vs $2 \mathrm{mmol} / 1$ glucose

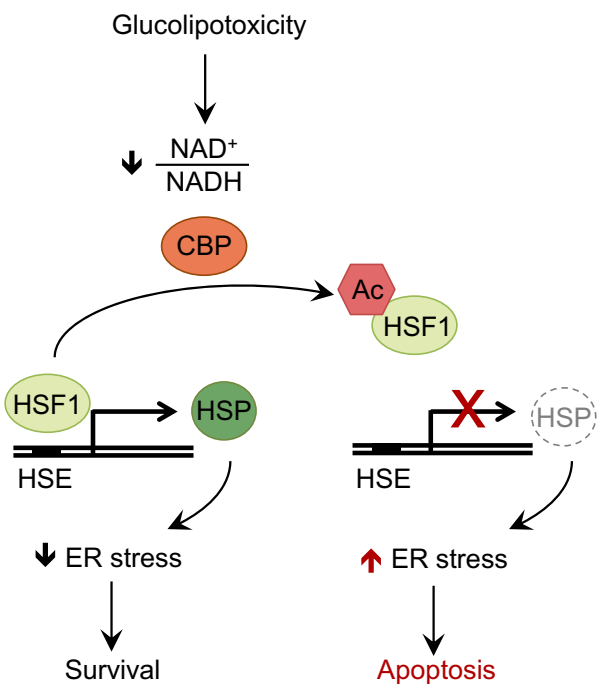

Fig. 7 Schematic representation of the role of HSF1 in glucolipotoxicityinduced ER stress and apoptosis. In the basal state, HSF1 binds to its responsive element, HSE, to stimulate HSP expression. Chronic exposure to elevated levels of glucose and NEFA causes acetylation (Ac) of HSF1, which acts as a molecular brake to inhibit its DNA binding activity. This blunts HSP expression, thereby facilitating ER stress and apoptosis

being developed and tested in models of type 2 diabetes. One of these agents, BGP-15, has been shown to improve insulin sensitivity and is being tested in phase 2 clinical trials $[14,27]$. A putative role for HSF1 in the maintenance of functional beta cell mass would complement its action on insulin sensitivity and, to our knowledge, has never been investigated.

We herein show that HSF 1 transcriptional activity is inhibited in pancreatic beta cells exposed to glucolipotoxicity. Indeed, upon glucolipotoxic stress, HSF1 undergoes acetylation at a key regulatory residue (lysine K80) located in its DNA binding domain [12]. Acetylation of K80 attenuates the DNA binding activity of HSF1, thereby decreasing expression of molecular chaperones and precipitating beta cell demise. Importantly, HSF1 inhibition is necessary for glucolipotoxicity-induced beta cell apoptosis since restoration of HSF1 activity or expression of a non-acetylatable HSF1 mutant prevented glucolipotoxicity-induced CHOP expression and cell death. Consistent with this, the expression of $H s f l$ and some of its canonical transcriptional targets were altered in islets isolated from diabetic GK rats, a model of spontaneous type 2 diabetes characterised by progressive loss of functional beta cell mass. Altogether, these findings unravel a novel role for HSF1 in the regulation of beta cell mass.

HSF1 acetylation has been shown to be regulated by the antagonistic actions of the acetyltransferase CBP and the protein deacetylase SIRT1 [12], the activity of which is regulated by cellular $\mathrm{NAD}^{+} / \mathrm{NADH}$ levels. Indeed, upon heat shock, SIRT1 was found to deacetylate HSF1, upregulating its activity and orchestrating the transcription of heat shock response. Using co-immunoprecipitation experiments, we herein demonstrated increased CBP binding to HSF1 upon glucolipotoxicity 
exposure. This interaction was disrupted in mutants harbouring a K80 mutation, confirming the importance of this key residue. Consistently, we showed that glucolipotoxicity-mediated HSF1 acetylation correlates with decreased $\mathrm{NAD}^{+} / \mathrm{NADH}$ levels. We also found that pharmacological inhibition of SIRT1 with nicotinamide decreased HSF1 activity (not shown). The regulation of HSF1 by SIRT1 could be of clinical significance since the therapeutic potential of SIRT1 is being scrutinised in type 2 diabetes and age-related diseases [28, 29].

Surprisingly, the transcriptional targets of HSF1 remain elusive. A few studies have combined expression profiling and chromatin immunoprecipitation to identify the transcriptional targets of HSF1 [24, 25] but our comprehension remains incomplete because binding of HSF1 to predicted HSEs is not sufficient to confer expression. In addition, HSF1 does not regulate the induction of every heat shock responsive gene. Moreover, our definition of the HSE is incomplete and an alternative binding element consisting of nGAAn repeats has been found in Saccharomyces cerevisiae $[24,25]$. Be that as it may, several putative HSF1 target genes were found to be differentially expressed in human pancreatic islets exposed to environmental stresses or disease. Indeed, in a previous genomic study, human islets exposed to elevated concentrations of palmitate displayed upregulation of several HSPs [30]. However, HSPA1A and HSPA6, the two specific isoforms investigated in our study, were not differentially expressed. Bugliani et al studied the transcriptome of pancreatic islets from individuals with type 2 diabetes and demonstrated downregulation of several de-ubiquitinating enzymes of the USP family [31]. Finally, genome-wide association studies have linked the homeodomain transcription factor haematopoietically expressed homeobox (HHEX) to type 2 diabetes, and variations within the HHEX gene are associated with decreased insulin secretion [32]. Collectively, these observations suggest that HSF1 could play a pivotal role in beta cells and deserves scrutiny as a potential therapeutic target.

In conclusion, we show that glucolipotoxicity causes acetylation of HSF1, thus inhibiting its transcriptional activity. We also show that HSF1 inhibition is necessary for glucolipotoxicityinduced beta cell apoptosis, since restoration of HSF1 activity prevents beta cell apoptosis. Our study thereby identifies a novel mechanism by which glucolipotoxicity causes ER stress and apoptosis, and characterises HSF1 for the first time as a potential target for the preservation of beta cell mass and function.

Acknowledgements We thank Y. W. Wang (University of Alberta, Edmonton, AB, Canada) who provided technical support.

Data availability All data generated and analysed during this study are included in this published article. The datasets generated during the current study are available from the corresponding author on reasonable request.
Funding IP was supported through Fellowships from the Natural Sciences and Engineering Research Council of Canada, and Alberta Diabetes Foundation. JB holds a Canadian Institutes of Health Research (CIHR) New Investigator Award. This work was supported by CIHR (JB).

Duality of interest The authors declare that there is no duality of interest associated with this article.

Contribution statement All authors contributed to experimental design, data acquisition and analysis, and writing the manuscript. All authors approved the final version. JB is the guarantor of this work.

\section{References}

1. Prentki M, Nolan CJ (2006) Islet beta cell failure in type 2 diabetes. J Clin Invest 116:1802-1812

2. Meier JJ, Breuer TG, Bonadonna RC et al (2012) Pancreatic diabetes manifests when beta cell area declines by approximately $65 \%$ in humans. Diabetologia 55:1346-1354

3. Butler AE, Janson J, Bonner-Weir S, Ritzel R, Rizza RA, Butler PC (2003) $\beta$-Cell deficit and increased $\beta$-cell apoptosis in humans with type 2 diabetes. Diabetes 52:102-110

4. Rhodes CJ (2005) Type 2 diabetes-a matter of $\beta$-cell life and death? Science 307:380-384

5. El-Assaad W, Buteau J, Peyot ML et al (2003) Saturated fatty acids synergize with elevated glucose to cause pancreatic beta-cell death. Endocrinology 144:4154-4163

6. El-Assaad W, Joly E, Barbeau A et al (2010) Glucolipotoxicity alters lipid partitioning and causes mitochondrial dysfunction, cholesterol, and ceramide deposition and reactive oxygen species production in INS832/13 ss-cells. Endocrinology 151:3061-3073

7. Karaskov E, Scott C, Zhang L, Teodoro T, Ravazzola M, Volchuk A (2006) Chronic palmitate but not oleate exposure induces endoplasmic reticulum stress, which may contribute to INS-1 pancreatic beta-cell apoptosis. Endocrinology 147:3398-3407

8. Cnop M, Ladriere L, Hekerman P et al (2007) Selective inhibition of eukaryotic translation initiation factor $2 \alpha$ dephosphorylation potentiates fatty acid-induced endoplasmic reticulum stress and causes pancreatic $\beta$-cell dysfunction and apoptosis. J Biol Chem 282: 3989-3997

9. Volchuk A, Ron D (2010) The endoplasmic reticulum stress response in the pancreatic $\beta$-cell. Diabetes Obes Metab 12(Suppl 2):48-57

10. Mathew A, Morimoto RI (1998) Role of the heat-shock response in the life and death of proteins. Ann N Y Acad Sci 851:99-111

11. Vihervaara A, Sistonen L (2014) HSF1 at a glance. J Cell Sci 127: 261-266

12. Westerheide SD, Anckar J, Stevens SM Jr, Sistonen L, Morimoto RI (2009) Stress-inducible regulation of heat shock factor 1 by the deacetylase SIRT1. Science 323:1063-1066

13. Kurucz I, Morva A, Vaag A et al (2002) Decreased expression of heat shock protein 72 in skeletal muscle of patients with type 2 diabetes correlates with insulin resistance. Diabetes 51:1102-1109

14. Chung J, Nguyen AK, Henstridge DC et al (2008) HSP72 protects against obesity-induced insulin resistance. Proc Natl Acad Sci U S A 105:1739-1744

15. Henry C, Close AF, Buteau J (2014) A critical role for the neural zinc factor ST18 in pancreatic $\beta$-cell apoptosis. J Biol Chem 289: 8413-8419

16. Mosser DD, Theodorakis NG, Morimoto RI (1988) Coordinate changes in heat shock element-binding activity and HSP70 gene transcription rates in human cells. Mol Cell Biol 8:4736-4744 
17. Bastien-Dionne PO, Valenti L, Kon N, Gu W, Buteau J (2011) Glucagon-like peptide 1 inhibits the sirtuin deacetylase SirT1 to stimulate pancreatic $\beta$-cell mass expansion. Diabetes 60:32173222

18. Buteau J, El-Assaad W, Rhodes CJ, Rosenberg L, Joly E, Prentki M (2004) Glucagon-like peptide-1 prevents beta cell glucolipotoxicity. Diabetologia 47:806-815

19. Cunha DA, Hekerman P, Ladriere L et al (2008) Initiation and execution of lipotoxic ER stress in pancreatic $\beta$-cells. J Cell Sci 121:2308-2318

20. Laybutt DR, Preston AM, Akerfeldt MC et al (2007) Endoplasmic reticulum stress contributes to beta cell apoptosis in type 2 diabetes. Diabetologia 50:752-763

21. Wang H, Kouri G, Wollheim CB (2005) ER stress and SREBP-1 activation are implicated in $\beta$-cell glucolipotoxicity. J Cell Sci 118: 3905-3915

22. Akerfelt M, Morimoto RI, Sistonen L (2010) Heat shock factors: integrators of cell stress, development and lifespan. Nat Rev Mol Cell Biol 11:545-555

23. Portha B, Giroix MH, Tourrel-Cuzin C, Le-Stunff H, Movassat J (2012) The GK rat: a prototype for the study of non-overweight type 2 diabetes. Methods Mol Biol 933:125-159

24. Page TJ, Sikder D, Yang L et al (2006) Genome-wide analysis of human HSF1 signaling reveals a transcriptional program linked to cellular adaptation and survival. Mol BioSyst 2:627-639
25. Trinklein ND, Murray JI, Hartman SJ, Botstein D, Myers RM (2004) The role of heat shock transcription factor 1 in the genome-wide regulation of the mammalian heat shock response. Mol Biol Cell 15:1254-1261

26. Henstridge DC, Whitham M, Febbraio MA (2014) Chaperoning to the metabolic party: the emerging therapeutic role of heat-shock proteins in obesity and type 2 diabetes. Mol Metab 3:781-793

27. Henstridge DC, Bruce CR, Drew BG et al (2014) Activating HSP72 in rodent skeletal muscle increases mitochondrial number and oxidative capacity and decreases insulin resistance. Diabetes 63:1881-1894

28. Hubbard BP, Sinclair DA (2014) Small molecule SIRT1 activators for the treatment of aging and age-related diseases. Trends Pharmacol Sci 35:146-154

29. Kitada M, Koya D (2013) SIRT1 in type 2 diabetes: mechanisms and therapeutic potential. Diabetes Metab J 37:315-325

30. Cnop M, Abdulkarim B, Bottu G et al (2014) RNA sequencing identifies dysregulation of the human pancreatic islet transcriptome by the saturated fatty acid palmitate. Diabetes 63:1978-1993

31. Bugliani M, Liechti R, Cheon H et al (2013) Microarray analysis of isolated human islet transcriptome in type 2 diabetes and the role of the ubiquitin-proteasome system in pancreatic beta cell dysfunction. Mol Cell Endocrinol 367:1-10

32. Pascoe L, Tura A, Patel SK et al (2007) Common variants of the novel type 2 diabetes genes CDKAL1 and HHEXIIDE are associated with decreased pancreatic beta-cell function. Diabetes 56:3101-3104 